\title{
Experimental and Theoretical Study on the Olefin Metathesis of Alkenyl Baylis-Hillman Adducts Using Second Generation Grubbs Catalyst
}

\author{
Mi Jung Lee ${ }^{\dagger}$, Ka Young Lee ${ }^{\dagger}$, Jin Yong Lee ${ }^{*,}$, and Jae Nyoung Kim*, ${ }^{\dagger}$ \\ ${ }^{\dagger}$ Department of Chemistry and Institute of Basic Science, Chonnam National University, \\ 300 Yongbong-Dong, Bugku, Gwangju 500-757, Korea \\ Institute for Condensed Matter Theory and Department of Chemistry, Chonnam \\ National University, 300 Yongbong-Dong, Bugku, Gwangju 500-757, Korea
}

\section{Supporting Information}

\section{Contents}

1. Synthetic details and characterization data.

2. Optimized structures of styrene, $\mathrm{PCy}_{3}$, and $\mathbf{3 a}$ (Figure S1).

3. Optimized structures of $\mathbf{4 a}, \mathbf{5 a}, \mathbf{6 a}, 7 \mathbf{a}$, and $\mathbf{8 a}$ (Figure S2).

4. Optimized structures of four different configurations trans-trans, trans-cis, cistrans, and cis-cis of 7a (Figure S3).

5. Optimized structures of metallacyclobutane intermediates for RCM and CM process for the reaction of isopentenyl methacrylate using second-generation Grubbs catalyst (Figure S4). 


\section{Synthetic details and characterization data}

Synthesis of starting materials 3a and 3b: The Baylis-Hillman adduct $\mathbf{1}$ was prepared in $66 \%$ yield from the reaction of benzaldehyde and methyl acrylate in the presence of DABCO. To a stirred solution of $1(595 \mathrm{mg}, 3.1 \mathrm{mmol})$ in aq. $\mathrm{MeOH}(4 \mathrm{~mL}, 2: 1)$ was added $\mathrm{KOH}(265 \mathrm{mg}, 4.0 \mathrm{mmol}$ ) and stirred at room temperature for $10 \mathrm{~h}$. After the usual aqueous workup and flash column chromatography (hexanes/ether, 3:1) we obtained 2 (316 mg, 57\%) as colorless oil. 2: ${ }^{1} \mathrm{H}$ NMR $\left(\mathrm{CDCl}_{3}\right) \delta 2.04(\mathrm{~s}, 1 \mathrm{H}), 5.54(\mathrm{~s}$, 1H), $5.94(\mathrm{~s}, 1 \mathrm{H}), 6.47(\mathrm{~s}, 1 \mathrm{H}), 7.25-7.36(\mathrm{~m}, 5 \mathrm{H})$. To a stirred mixture of $2(285 \mathrm{mg}$, $1.6 \mathrm{mmol})$ and 4-bromo-1-butene $(273 \mathrm{mg}, 2.0 \mathrm{mmol})$ in $\mathrm{CH}_{3} \mathrm{CN}(4 \mathrm{~mL})$ was added DBU (305 mg, $2 \mathrm{mmol}$ ). The reaction mixture was stirred at $50{ }^{\circ} \mathrm{C}$ for $12 \mathrm{~h}$. After the usual aqueous workup and flash column chromatography (hexanes/ether, 5:1) we obtained 3a (194 mg, 52\%) as colorless oil. IR (neat) $3471,1716,1273,1149 \mathrm{~cm}^{-1} ;{ }^{1} \mathrm{H}$ $\operatorname{NMR}\left(\mathrm{CDCl}_{3}\right) \delta 2.31-2.39(\mathrm{~m}, 2 \mathrm{H}), 3.10(\mathrm{~d}, J=5.7 \mathrm{~Hz}, 1 \mathrm{H}), 4.15(\mathrm{t}, J=6.6 \mathrm{~Hz}, 2 \mathrm{H})$, 5.02-5.10 (m, 2H), $5.53(\mathrm{~d}, J=5.7 \mathrm{~Hz}, 1 \mathrm{H}), 5.64-5.78(\mathrm{~m}, 1 \mathrm{H}), 5.82(\mathrm{t}, J=1.2 \mathrm{~Hz}, 1 \mathrm{H})$,

$6.34(\mathrm{t}, J=1.2 \mathrm{~Hz}, 1 \mathrm{H}), 7.25-7.38(\mathrm{~m}, 5 \mathrm{H}) ;{ }^{13} \mathrm{C} \mathrm{NMR}\left(\mathrm{CDCl}_{3}\right) \delta 32.84,63.84,73.19$, $117.35,126.08,126.54,127.74,128.35,133.76,141.25,142.02$, 166.15. Similarly 3b was synthesized from the reaction of 2 and allyl bromide in $75 \%$ yield. IR (neat) 3448, 1716, 1273, $1146 \mathrm{~cm}^{-1} ;{ }^{1} \mathrm{H}$ NMR $\left(\mathrm{CDCl}_{3}\right) \delta 3.02(\mathrm{~d}, J=5.7 \mathrm{~Hz}, 1 \mathrm{H}), 4.61(\mathrm{dt}, J=5.4$ and 1.5 Hz, 2H), 5.18-5.29 (m, 2H), $5.57(\mathrm{~d}, J=5.7 \mathrm{~Hz}, 1 \mathrm{H}), 5.81-5.94(\mathrm{~m}, 1 \mathrm{H}), 5.86(\mathrm{~s}$, 1H), $6.38(\mathrm{~s}, 1 \mathrm{H}), 7.26-7.40(\mathrm{~m}, 5 \mathrm{H}) ;{ }^{13} \mathrm{C} \mathrm{NMR}\left(\mathrm{CDCl}_{3}\right) \delta 65.45,73.24,118.38,126.25$, $126.58,127.83,128.41,131.67,141.18,141.94,165.91$.

The reaction of $\mathbf{3 a}$ with Grubbs catalyst: To a stirred solution of 3a (151 mg, 0.65 $\mathrm{mmol})$ in dry toluene $(50 \mathrm{~mL})$ was added dropwise a solution of catalyst 4 (77 mg, 14 mol\%) in toluene $(50 \mathrm{~mL})$ during $20 \mathrm{~h}$ at $70-80{ }^{\circ} \mathrm{C}$. After addition of the catalyst, the 
reaction mixture was further stirred for $20 \mathrm{~h}$. After removal of the solvent and flash column chromatography (hexanes/ether, 5:1) we obtained 10a (15 mg, 8\%) and 11a (65 mg, 46\%) as clear oils. 10a: IR (neat) $3479,1716,1269,1153 \mathrm{~cm}^{-1} ;{ }^{1} \mathrm{H}$ NMR $\left(\mathrm{CDCl}_{3}\right) \delta$ 2.41-2.49 (m, 2H), $2.87(\mathrm{~d}, J=5.7 \mathrm{~Hz}, 1 \mathrm{H}), 4.17(\mathrm{t}, J=6.6 \mathrm{~Hz}, 2 \mathrm{H}), 5.49(\mathrm{~d}, J=5.7 \mathrm{~Hz}$, 1H), 5.74-5.80 (m, 1H), $6.02(\mathrm{dt}, J=15.9$ and $6.9 \mathrm{~Hz}, 1 \mathrm{H}), 6.29(\mathrm{~s}, 1 \mathrm{H}), 6.36(\mathrm{dt}, J=$ 15.9 and $1.2 \mathrm{~Hz}, 1 \mathrm{H}), 7.13-7.31(\mathrm{~m}, 10 \mathrm{H}) ;{ }^{13} \mathrm{C} \mathrm{NMR}\left(\mathrm{CDCl}_{3}\right) \delta 32.24,64.13,73.36$, $125.34,126.10,126.26,126.55,127.34,127.83,128.43,128.53,132.60,137.13,141.27$, 141.98, 166.22. 11a: IR (neat) 3448, 1712, 1273, $1153 \mathrm{~cm}^{-1} ;{ }^{1} \mathrm{H}$ NMR $\left(\mathrm{CDCl}_{3}\right) \delta 2.24-$ $2.32(\mathrm{~m}, 4 \mathrm{H}), 3.08(\mathrm{~d}, J=5.7 \mathrm{~Hz}, 2 \mathrm{H}), 4.10(\mathrm{td}, J=6.6$ and $1.5 \mathrm{~Hz}, 4 \mathrm{H}), 5.36-5.39(\mathrm{~m}$, 2H), $5.54(\mathrm{~d}, J=5.7 \mathrm{~Hz}, 2 \mathrm{H}), 5.81-5.83(\mathrm{~m}, 2 \mathrm{H}), 6.31-6.33(\mathrm{~m}, 2 \mathrm{H}), 7.25-7.38(\mathrm{~m}$, $10 \mathrm{H}) ;{ }^{13} \mathrm{C} \mathrm{NMR}\left(\mathrm{CDCl}_{3}\right) \delta 31.75,64.11,73.20,126.16,126.55,127.80,128.35,128.40$, 141.26, 142.02, 166.13. Similarly we obtained $\mathbf{1 1 b}$ from the reaction of $\mathbf{3 b}$ with Grubbs catalyst. 11b: ${ }^{1} \mathrm{H} \mathrm{NMR}\left(\mathrm{CDCl}_{3}\right) \delta 2.93(\mathrm{~d}, J=5.4 \mathrm{~Hz}, 2 \mathrm{H}), 4.59(\mathrm{~m}, 4 \mathrm{H}), 5.57(\mathrm{~d}, J=$ $5.4 \mathrm{~Hz}, 2 \mathrm{H}), 5.68-5.70(\mathrm{~m}, 2 \mathrm{H}), 5.88(\mathrm{t}, J=1.2 \mathrm{~Hz}, 2 \mathrm{H}), 6.37(\mathrm{~s}, 2 \mathrm{H}), 7.26-7.39(\mathrm{~m}$, $10 \mathrm{H}) ;{ }^{13} \mathrm{C} \mathrm{NMR}\left(\mathrm{CDCl}_{3}\right) \delta 64.07,73.24,126.37,126.60,127.64,127.89,128.45$, $141.16,141.85,165.78$.

We observed low intensity for the corresponding $\mathrm{M}^{+}$peak or no $\mathrm{M}^{+}$peak in some cases presumably due to the instability of the molecular cation radical species. Thus, we took LRMS spectra of the prepared compounds 3a, 3b, 10a, 11a, and 11b (EIMS, 70 eV) instead of HRMS.

3a: MS (70 eV) m/z (rel. intensity) 53 (100), 55 (76), 79 (96), 105 (65), 115 (55), 159

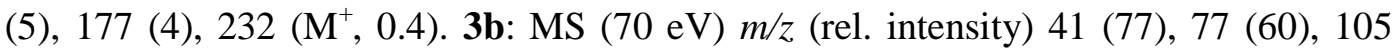
(100), 159 (32), 177 (15), 201 (0.7), 218 ( $\left.\mathrm{M}^{+}, 0.2\right)$. 10a: MS (70 eV) m/z (rel. intensity) 
43 (38), 55 (23), 77 (21), 91 (30), 105 (15), 115 (31), 130 (100), no $\mathrm{M}^{+}$. 11a: MS (70 eV) $\mathrm{m} / z$ (rel. intensity) 80 (100), 105 (81), 115 (59), 143 (18), 159 (11), 177 (6), 198 (0.4), 215 (1.2), 242 (1.0), 302 (1.2), $418(0.1)$, no $\mathrm{M}^{+}$. 11b: MS (70 eV) m/z (rel. intensity) 54 (55), 79 (100), 105 (58), 115 (62), 159 (8), 161 (6), no $\mathbf{M}^{+}$. 
Figure S1. Optimized structures of styrene, $\mathrm{PCy}_{3}$, and $\mathbf{3 a}$.

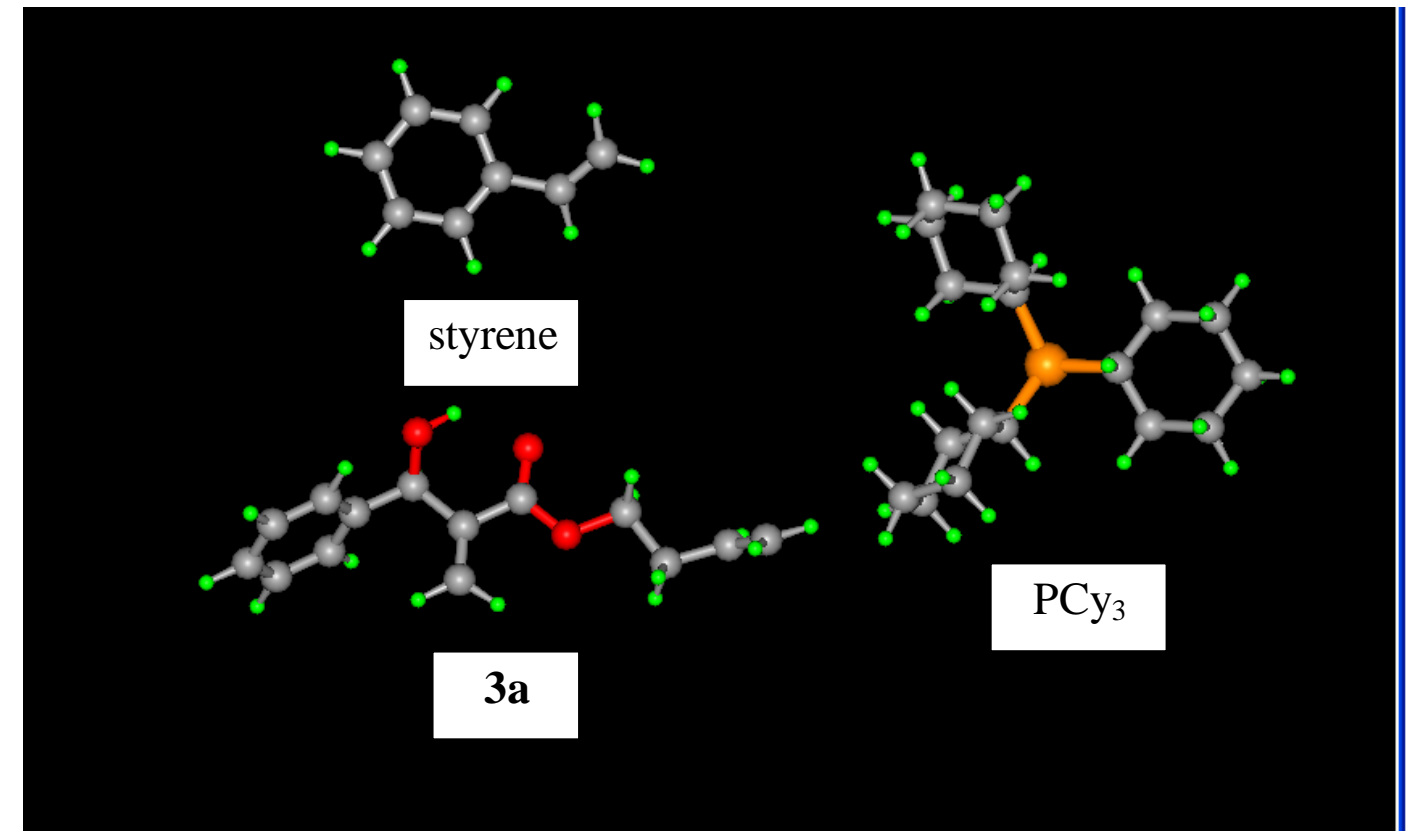


Figure S2. Optimized structures of $\mathbf{4 a}, \mathbf{5 a}, \mathbf{6 a}, 7 \mathbf{a}$, and $8 \mathbf{a}$.

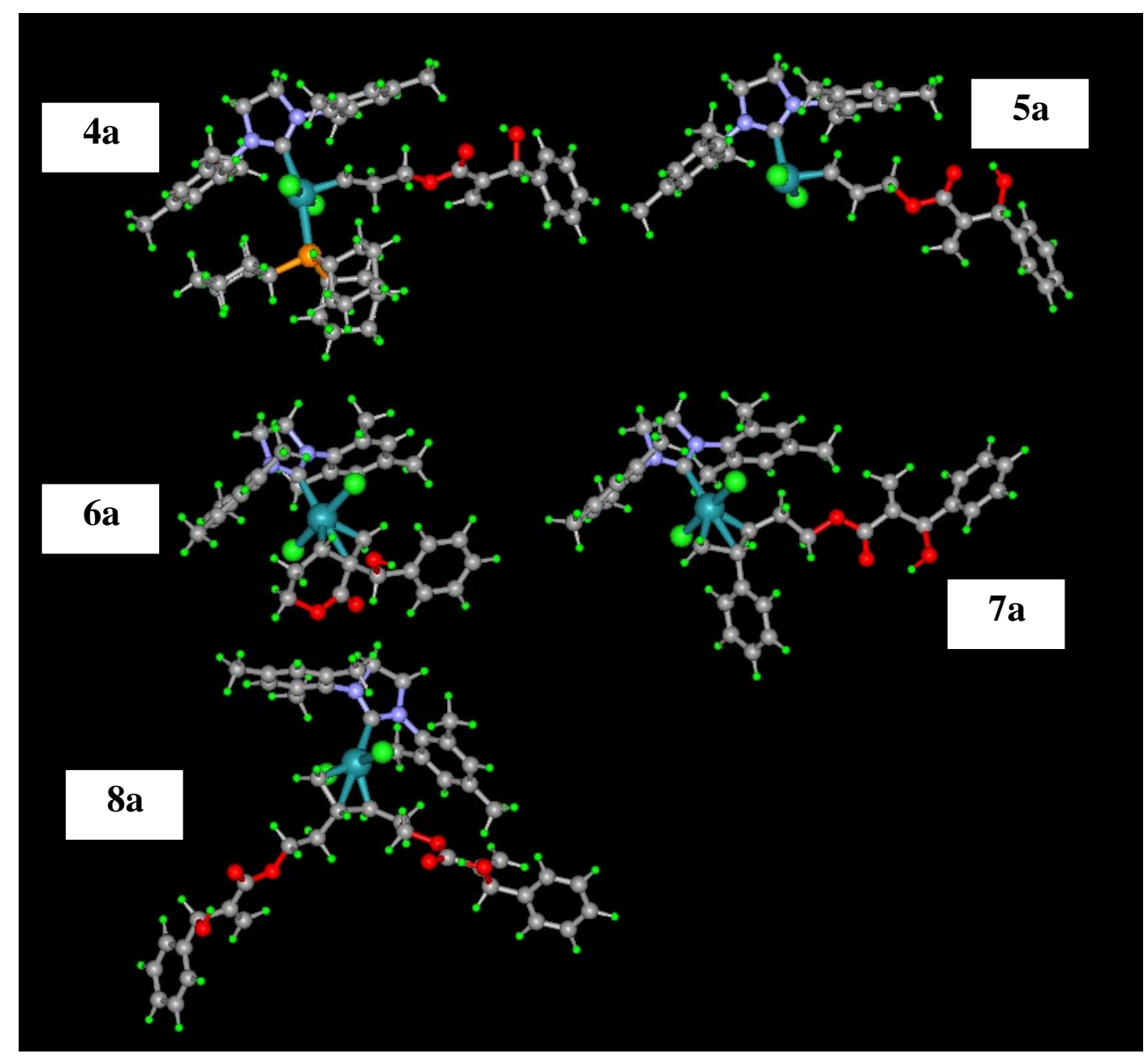


Figure S3. Optimized structures of four different configurations trans-trans, trans-cis, cis-trans, and cis-cis of 7a.

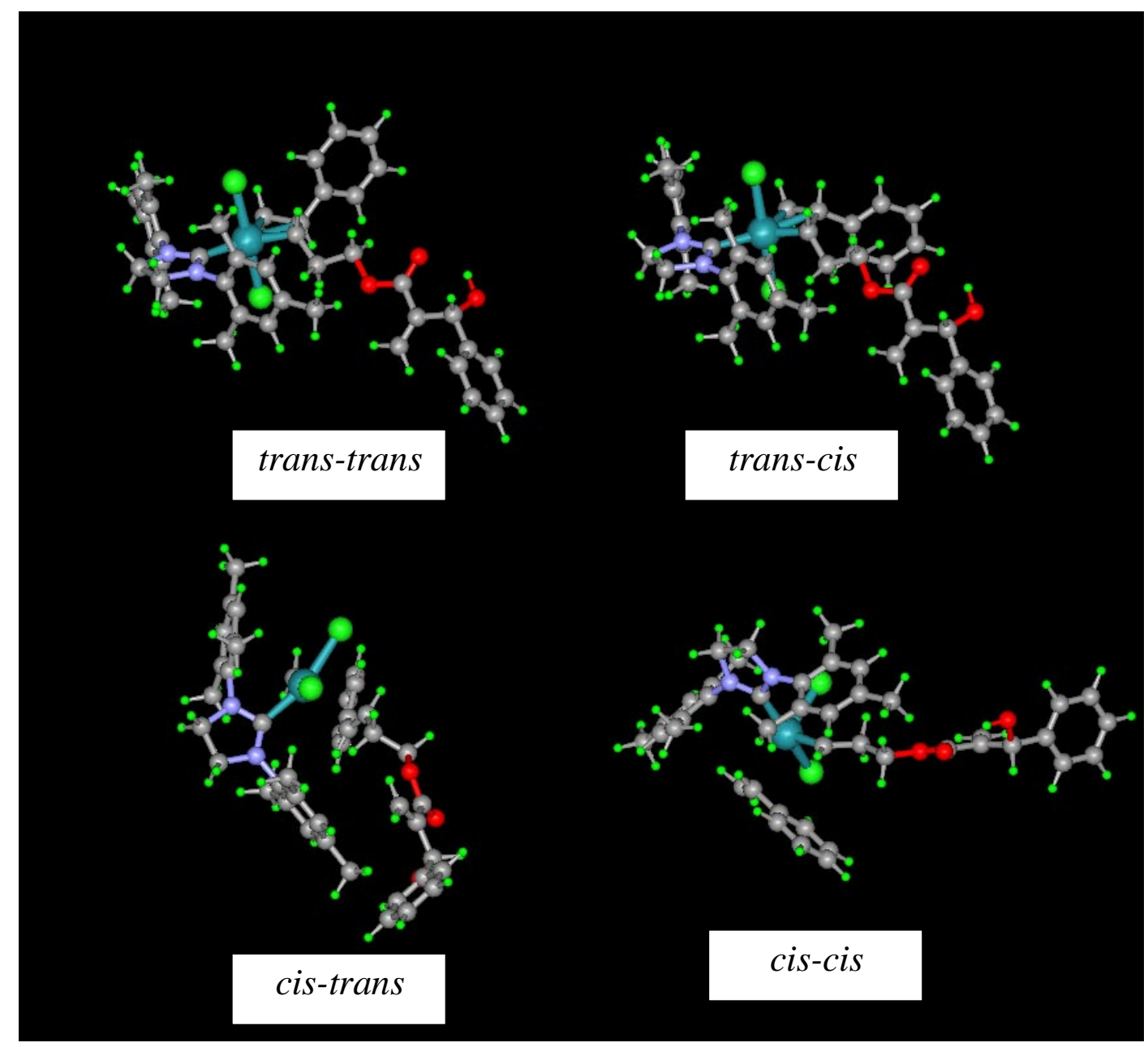


Figure S4. Optimized structures of metallacyclobutane intermediates for RCM and CM process for the reaction of isopentenyl methacrylate using second-generation Grubbs catalyst.

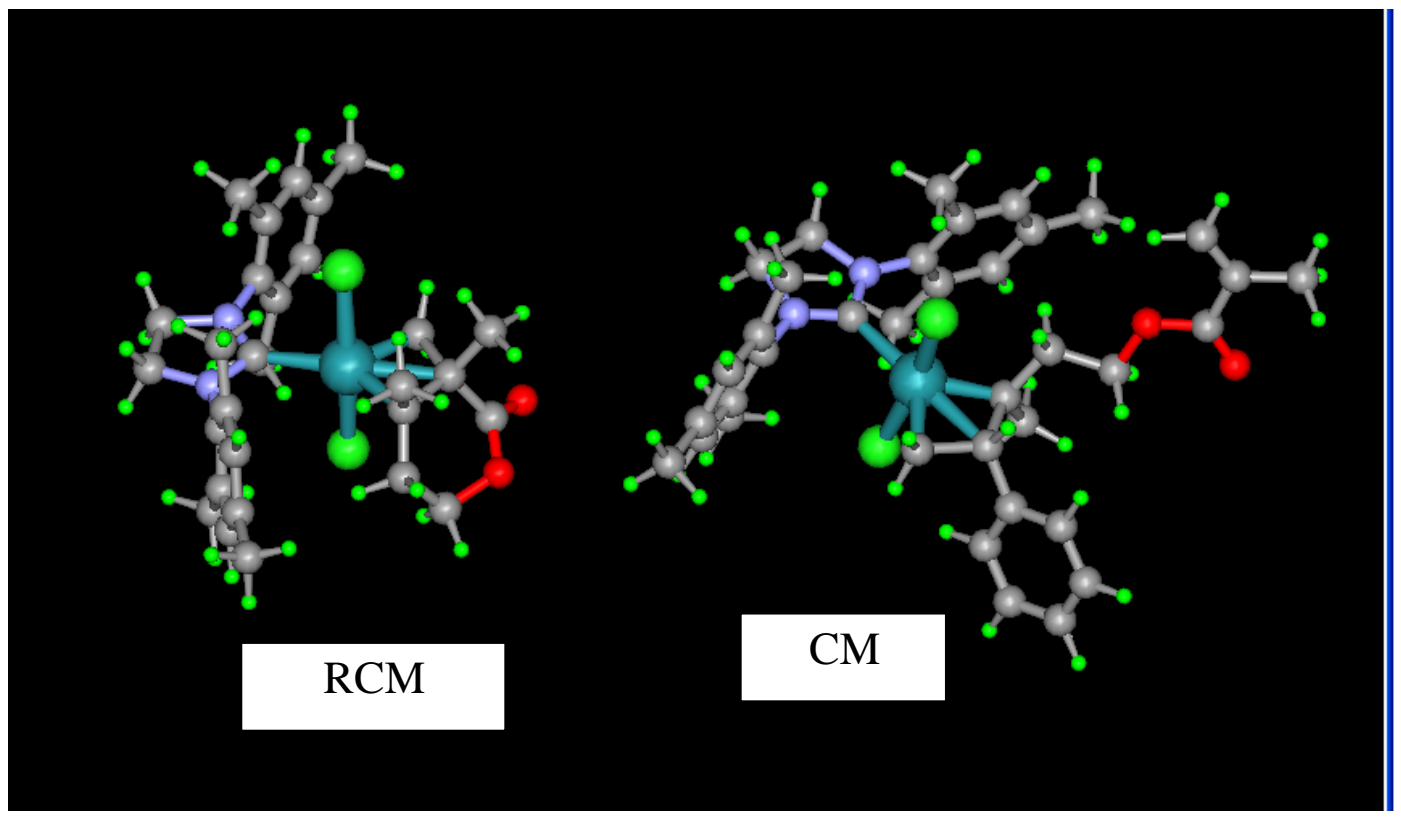

